\title{
Posttraumatic Subperiosteal Orbital Hematoma With Intracranial Hemorrhage in A Male Infant
}

\section{Yeon Jeong Lee}

Kangwon National University Hospital

Yungju Yoo ( $\nabla$ yyjuuuu@gmail.com )

Kangwon National University College of Medicine: Kangwon National University School of Medicine https://orcid.org/0000-0001-8045-0391

\section{Case Report}

Keywords: orbital hematoma, trauma, intracranial hemorrhage

Posted Date: January 19th, 2021

DOl: https://doi.org/10.21203/rs.3.rs-147575/v1

License: (1) This work is licensed under a Creative Commons Attribution 4.0 International License. Read Full License 


\section{Abstract}

Background: To the best of our knowledge, there has been no report of subperiosteal orbital hematoma combined with intracerebral hemorrhage in pediatric patients.

Case presentation: A healthy 9-month-old boy with left superior tarsal conjunctival prolapse was admitted to the emergency room after left periorbital blunt trauma. Computer tomography of the brain showed a left orbital roof fracture and subperiosteal orbital hematoma with acute intracerebral hemorrhage. He received emergency craniotomy. Three days after the craniotomy, the subperiosteal hematoma and proptosis increased. An anterior orbitotomy with orbital subperiosteal hematoma evacuation and drain placement were performed. Nine months after surgery, the left blepharoptosis and conjunctival prolapse improved and fix and follow were good in his both eyes. However, a new-onset intermittent exotropia and left hypotropia were detected.

Conclusions: Orbital subperiosteal hematoma must be considered in the differential diagnosis of acute unilateral proptosis after trauma. In infants and children, a thorough work-up to detect concomitant intracerebral hemorrhage is necessary. Prompt surgical removal of hematoma is recommended to prevent complications

\section{Background}

In children, subperiosteal orbital hematoma constitutes less than $1 \%$ of space-occupying lesions. Most commonly, an extraorbital hematoma co-exists or originates from a subgaleal hematoma [1]. To the best of our knowledge, there has been no report of subperiosteal orbital hematoma combined with intracerebral hemorrhage in pediatric patients. Herein, we describe a rare presentation of posttraumatic subperiosteal hematoma combined with intracerebral hemorrhage in a 9-month-old boy.

\section{Case Presentation}

A 9-month-old infant was referred with blepharoptosis and conjunctival prolapse of his left eye. A day back, the infant had accidently bumped his left eyebrow area against his grandmothers' elbow. The investigation to rule out the possibility of child abuse was conducted and turned out it was an accident. On initial examination, periorbital ecchymosis and upper tarsal conjunctival prolapse were noted in his left eye (Fig. 1). Blood coagulation test was normal. Computer tomography (CT) and magnetic resonance imaging of the brain demonstrated left intraorbital hematoma, left orbital roof fracture, and intracerebral hemorrhage involving the left frontal lobe (Fig. 1). The patient was referred to the neurosurgery department and received an emergent craniotomy with evacuation of the intracranial hemorrhage.

The proptosis and conjunctival prolapse in the left eye were progressed. An orbital CT performed on the third post-craniotomy day showed a significant increase in subperiosteal hematoma close to the roof (Fig. 1). Spontaneous regression was not expected due to significant increase of subperiosteal hematoma, and hence a second emergency surgery was performed to remove the subperiosteal 
hematoma. The subperiosteal space was entered through a superonasal orbital rim incision, and two drains were placed under the subperiosteum of the orbital roof. The intraocular pressure was $11.4 \mathrm{mmHg}$ before the surgery and $8.0 \mathrm{mmHg}$ before the surgery by rebound tonometry (iCare; Icare Finland Oy, Helsinki, Finland). Laboratory analysis of the drained fluid revealed a red blood cell density of $47,500 / \mathrm{mm}^{3}$, white blood cell density of $60 / \mathrm{mm}^{3}$ and a glucose level of $78 \mathrm{mg} / \mathrm{dl}$. The amount of drainage fluid reduced gradually, and the drains were removed two days after placement.

After hematoma evacuation, the proptosis and downward shift of the eyeball improved without any further intervention. On the fourteenth postsurgical day, fix and follow of the left eye was poor, but the left upper tarsal conjunctival prolapse had reduced. One hour of occlusion of the right eye was recommended to prevent stimulus deprivation amblyopia. After eight months following orbitotomy, the fix and follow of the left eye improved. The marginal reflex distance was $3 \mathrm{~mm}$ and $2 \mathrm{~mm}$ in the right and left eyes, respectively and 30 prism diopters of intermittent left exotropia and 10 prism diopters of left hypotropia by Krimsky test were detected (Fig. 1).

\section{Discussion And Conclusions}

Subperiosteal hematoma is a rare complication of periorbital injury and can evoke critical visual defects. The estimated incidence of intraorbital hematoma is $0.3 \%$, while orbital subperiosteal hematomas are less common [2]. In pediatrics, orbital subperiosteal hematoma accounts for less than $1 \%$ of spaceoccupying lesions [1]. There are reports of post-traumatic orbital subperiosteal hematoma in children; however, the youngest age reported is two years [3]. We report the successful management of subperiosteal orbital hematoma in an infant.

The orbital subperiosteal hematoma after trauma mostly occurs in pediatric patients [4]. A possible explanation is the weak adherence of periosteum to the orbital roof in young subjects [4]. Such hematomas can compress the optic nerve causing visual impairment that persists even after its evacuation [1]. In a patient managed conservatively, the soft tissue swelling resolved in five weeks, and the subperiosteal hematoma took a significantly longer duration of four months to resolve completely [5]. Chronic orbital subperiosteal hematomas can eventually turn into cholesterol granulomas through granulomatous reaction or bone lytic lesions [6]. Considering the above possible complications, timely surgical removal of the hematoma is recommended.

The management of intraorbital hematoma ranges from observation to needle aspiration or surgical evacuations. The treatment of choice, however, remains controversial. Many studies recommend early surgical interventions for rapid recovery and to prevent sequelae [7]. In contrast, other reports recommend observation to prevent surgical complications such as local infections and re-bleeding [8]. It is important to note that successful conservative treatment was reported in children older than ten years who were not in the critical period for vision development $[1,6,7]$. 
Both blepharoptosis and conjunctival prolapse interfere with the pupillary axis, resulting in deprivation amblyopia in children under seven years of age [9]. Especially, physicians must consider this complication in all children under two years of age. In children with conjunctival prolapse, the blockage of pupillary axis causes visual deprivation and stimulus deprivation amblyopia. This type of amblyopia develops in the cases of congenital ptosis and congenital cataract. The best corrected visual acuity of an infant with eyelid swelling who was not treated for a short duration of six days reduced to below 0.125 [10]. Also, stimulus deprivation amblyopia can be resistant to treatment, leading to poor visual prognosis.

The relationship between congenital ptosis and strabismus is well reported. The incidence of strabismus in simple congenital ptosis is approximately $9.9 \%$ and is higher compared to its incidence in the general population (1\% to $5 \%$ ) [9]. It is suggested that strabismus results from visual occlusion and disruption of binocularity by the ptotic eyelid [9]. Therefore, careful examinations are essential in patients presenting with ptosis after trauma.

In summary, we describe the case of an infant who suffered subperiosteal orbital hematoma with intracerebral hemorrhage. Thus, in infants presenting with subperiosteal hematoma, it is important to rule out a combined intracerebral lesion. Early intervention is crucial to prevent stimulus deprivation amblyopia and strabismus. Occlusion therapy for amblyopia and close follow-up are recommended for such children after the initial management.

\section{Declarations}

Ethics approval and consent to participate: This study adhered to the Declaration of Helsinki and the protocol was approved by the Institutional Review Board of Kangwon National University Hospital. The parental consent was obtained with a signed consent form.

Consent for publication: The guidance of patient provided written consent for publication of the patient data and accompanying images in this case report.

Availability of data and material: All data generated or analyzed during this study are included in this published article.

Competing interests: none.

Funding: This study was supported by 2020 Kangwon National University Hospital Grant. The funding body had no role in study design, data analysis, data interpretation, or manuscript writing.

Authors' contributions: YJY contributed to data acquisition, data analysis, and manuscript drafting. YJL contributed to design of the work and manuscript revision. All authors read and approved the final manuscript.

Acknowledgements: Not applicable. 


\section{References}

1. Yazici B, Gonen T: Posttraumatic subperiosteal hematomas of the orbit in children. Ophthalmic plastic and reconstructive surgery 2011;27:33-37.

2. Wood GD: Blindness following fracture of the zygomatic bone. The British journal of oral \& maxillofacial surgery 1986;24:12-16.

3. Singh M, Gautam Seth N, Zadeng Z, Kaur M, Gupta P: Clinico-radiological features and treatment outcomes in children with traumatic orbital subperiosteal hematoma. Journal of AAPOS : the official publication of the American Association for Pediatric Ophthalmology and Strabismus 2018;22:416$420 . e 413$.

4. Nakai K, Doi E, Kuriyama T, Tanaka Y: Spontaneous subperiosteal hematoma of the orbit. Surgical neurology 1983;20:100-102.

5. Ganesan K, Fabbroni G, Loukota R, Craggs L: Traumatic subperiosteal hematoma of the orbit: a report of 2 cases. Journal of oral and maxillofacial surgery : official journal of the American Association of Oral and Maxillofacial Surgeons 2008;66:1266-1269.

6. Bergin DJ, McCord CD, Dutton JJ, Garrett SN: Chronic hematic cyst of the orbit. Ophthalmic plastic and reconstructive surgery 1988;4:31-36.

7. Mikami T, Maegawa J, Kuroda MM, Yamamoto Y, Yasumura K: Subacute phase treatment of subperiosteal hematoma of the orbit with epidural hematoma in the frontal cranial fossa: case report. BMC ophthalmology 2012;12:18.

8. Wolter JR: Subperiosteal hematomas of the orbit in young males: a serious complication of trauma or surgery in the eye region. Journal of pediatric ophthalmology and strabismus 1979;16:291-296.

9. Griepentrog GJ, Mohney BG: Strabismus in childhood eyelid ptosis. American journal of ophthalmology 2014;158:208-210 e201.

10. Upendran MR, McLoone E: Delayed resolution of eyelid swelling in preseptal cellulitis in a child: beware of causing occlusion amblyopia. BMJ case reports 2013;2013.

\section{Figures}




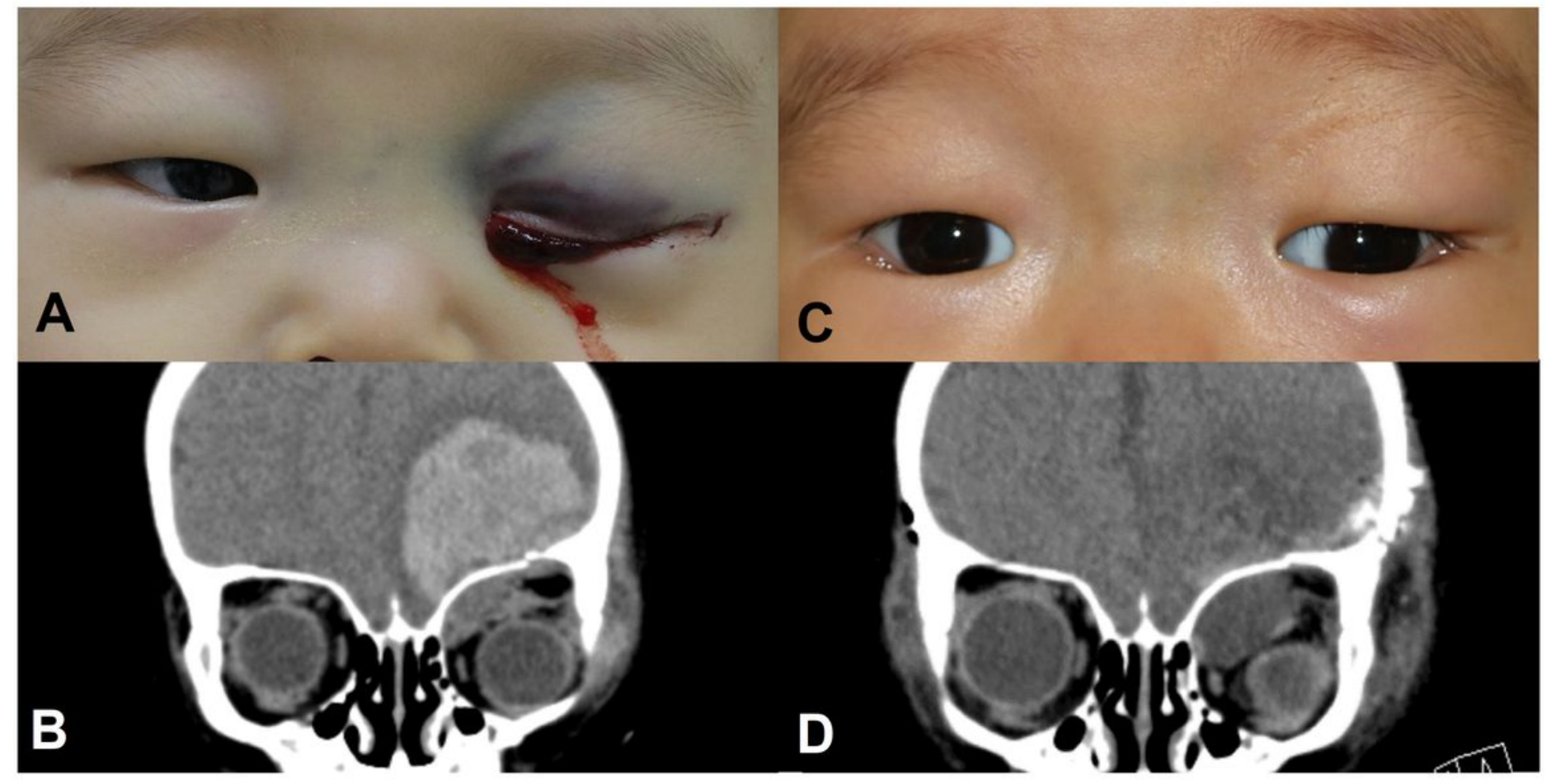

\section{Figure 1}

Eyelid photos and computed tomography. A, Clinical picture of the patient at initial examination. Left periorbital ecchymosis and mild superior tarsal conjunctival prolapse were noted. B, Computed tomography (CT) at initial examination. The coronal view of CT brain demonstrated left orbital roof fracture (arrow) and intracerebral hemorrhage (asterix) in the left frontal lobe. C, Clinical picture of the patient at nine months after surgical treatment. The marginal reflex distance was $3 \mathrm{~mm}$ in the right eye and $2 \mathrm{~mm}$ in the left eye. Thirty prism diopters of intermittent left exotropia and 10 prism diopters of left hypotropia by Krimsky test were detected. D, Repeat CT three days after the initial craniotomy. The coronal view of orbital CT showed markedly increased subperiosteal hematoma located in the left superior orbital roof. 